\title{
MARTIN BOUNDARIES OF DENJOY DOMAINS
}

\author{
SHIGEO SEGAWA
}

(Communicated by Paul S. Muhly)

\begin{abstract}
Let $E(\subset \hat{\mathbf{C}})$ be a compact set in the real axis. It is shown that there exists an $E$ with zero linear measure such that Martin compactification of the domain $\hat{\mathbf{C}}-E$ is not homeomorphic to $\hat{\mathbf{C}}$. Moreover, it is shown that if for some $\lambda>\frac{1}{2}$

$$
\frac{\left|E^{c} \cap[-t, t]\right|}{t}=O\left(\left(\frac{1}{\log t^{-1}}\right)^{\lambda}\right) \quad(t \rightarrow 0),
$$

the set of minimal Martin boundary points of $\hat{\mathbf{C}}-E$ 'over 0' consists of two points. This assertion is not valid for $\lambda=\frac{1}{2}$.
\end{abstract}

Consider a domain $D$ in $\hat{\mathbf{C}}=\mathbf{C} \cup\{\infty\}$ such that $\hat{\mathbf{C}}-D \subset \hat{\mathbf{R}}=\mathbf{R} \cup\{\infty\}$. Such a domain $D$ is said to be a Denjoy domain (cf. Garnett and Jones [4]). For $p \in E=\partial D$, let $P_{p}=P_{p}(D)$ be the class of positive harmonic functions on $D$ which are bounded except for any neighborhood of $p$, and have vanishing boundary values at every regular point of $E$ except $p$. Denote by $\operatorname{dim} P_{p}$ the cardinal number of the set of minimal functions $h$ in $P_{p}$ satisfying the normalized condition $h(a)=1$, $a \in D$. In terms of Martin compactification, $\operatorname{dim} P_{p}$ means the cardinal number of the set of minimal boundary points 'over $p$ '. It is easily seen that $\operatorname{dim} P_{p} \geq 1$ for every $p \in E$ (cf. e.g. Benedicks [2]). Ancona [1] and Benedicks [2], independently, showed that $\operatorname{dim} P_{p} \leq 2$ for every $p \in E$. Also, Maitani showed that if $\operatorname{dim} P_{p}=1$ for every $p \in E$ (in this case, the Martin compactification $D^{*}$ of $D$ is homeomorphic to $\bar{D}$, the closure of $D$ in $\hat{\mathbf{C}}$ ) the linear measure $|E|$ of $E$ is zero.

In this paper, applying the Benedicks criterion in [2], we shall show that the converse of the above Maitani result is invalid ( $\S 1)$, and study the cardinal number $\operatorname{dim} P_{p}$ for $p$ which is a point of density of $E(\S 2)$.

The author would like to express his sincere thanks to Professors M. Nakai, F. Maitani, and T. Murai for their valuable discussions with him, and also to Professor Y. Komatu for his helpful suggestion to the proof of Theorem 4.

1. We start by restating Maitani's result in the introduction.

ThEOREM 1. Let $D$ be a Denjoy domain. Suppose that $\operatorname{dim} P_{p}(D)=1$ for every $p \in E=\partial D$. Then the linear measure $|E|$ of $E$ is zero.

Since the above result was unpublished, we give the proof for the sake of completeness.

If $D$ is of null boundary, the capacity of $E$ is zero, and hence $|E|=0$. Thus we may assume that there exists the Green's function $g$ on $D$. Choose a point

Received by the editors January 29, 1987.

1980 Mathematics Subject Classification (1985 Revision). Primary 30F25, 31A05. 
$a \in D \cap \mathbf{R}$. By the assumption, the limit

$$
\lim _{\varsigma \rightarrow p} \frac{g(z, \varsigma)}{g(a, \zeta)} \quad\left(=k_{p}(z)\right)
$$

exists for every $p \in E$. From the fact that $g(a, \bar{\zeta})=g(a, \zeta)$, it follows that

$$
k_{p}(\bar{z})=\lim _{\zeta \rightarrow p} \frac{g(\bar{z}, \varsigma)}{g(a, \zeta)}=\lim _{\bar{\zeta} \rightarrow p} \frac{g(z, \bar{\zeta})}{g(a, \bar{\zeta})}=k_{p}(z) .
$$

Hence, by general Martin theory (here, we identify $E$ to the Martin boundary of $D)$, it is seen that

$$
h(\bar{z})=\int_{E} k_{p}(\bar{z}) d \mu_{h}(p)=\int_{E} k_{p}(z) d \mu_{h}(p)=h(z)
$$

for every positive harmonic function $h$ on $D$, where $\mu_{h}$ is a positive measure on $E$. This implies that $u(\bar{z})=u(z)$ for every bounded harmonic function $u$ on $D$, and hence it is easily verified that $|E|=0$.

Here we recall Benedicks' result in [2]. Let $E$ be a compact subset of $\hat{\mathbf{R}}=$ $\mathbf{R} \cup\{\infty\}$ containing $\infty$. Denote by $Q(t, r), t \in \mathbf{R}$, the square $\{\xi+i \eta ;|\xi-t|<r / 2$, $|\eta|<r / 2\}$. For an arbitrarily fixed $\alpha$ in the interval $(0,1)$ and every $x$ in $\mathbf{R}$, let $\beta_{x}(\cdot)=\beta_{x}(\cdot ; E, \alpha)$ be the solution of Dirichlet problem on $Q(x, \alpha|x|)-E$ for boundary values $\beta_{x}=1$ on $\partial Q(x, \alpha|x|)$ and $\beta_{x}=0$ on $E \cap Q(x, \alpha|x|)$. Then, Benedicks showed the following:

BENEDICKS' CRITERION I. For every $\alpha$ with $0<\alpha<1$,

$$
\begin{array}{ll}
\operatorname{dim} P_{\infty}(\hat{\mathbf{C}}-E)=1 \quad & \text { if and only if } \quad \int_{|x| \geq 1} \frac{\beta_{x}(x)}{|x|} d x=\infty, \\
\operatorname{dim} P_{\infty}(\widehat{\mathbf{C}}-E)=2 & \text { if and only if } \quad \int_{|x| \geq 1} \frac{\beta_{x}(x)}{|x|} d x<\infty .
\end{array}
$$

We are in the stage to prove the following Set

THEOREM 2. Let $E_{0}$ be a closed set in the interval $\left[-\frac{1}{2}, \frac{1}{2}\right]$ of positive capacity.

and

$$
E_{n}=E_{0}+n=\left\{x+n ; x \in E_{0}\right\} \quad(n \in \mathbf{Z})
$$

$$
E=\left(\bigcup_{n=-\infty}^{\infty} E_{n}\right) \cup\{\infty\} .
$$

Then, for the Denjoy domain $D=\widehat{\mathbf{C}}-E, \operatorname{dim} P_{\infty}(D)=2$.

Immediately, Theorem 2 shows that the converse of Theorem 1 is invalid.

LEMMA 3. Let $E$ be as in Theorem 2 and $h_{n}(n \in \mathbf{N})$ be the solution of Dirichlet problem on $Q\left(0,3^{n}\right)-E$ for boundary values $h_{n}=1$ on $\partial Q\left(0,3^{n}\right)$ and $h_{n}=0$ on $E \cap Q\left(0,3^{n}\right)$. Then, there exists a constant $c$ in $(0,1)$ such that

$$
h_{n}(x) \leq c^{n} \quad\left(x \in\left[-\frac{1}{2}, \frac{1}{2}\right]\right),
$$

where $c$ is independent of $n$ and $x$.

PROOF. Set $c_{1}=\sup _{x \in[-1 / 2,1 / 2]} h_{1}(x)$. Then $0<c_{1}<1$ and

$$
h_{n}(x) \leq c_{1} \quad\left(x \in\left[-3^{n-1} / 2,3^{n-1} / 2\right]\right) .
$$


Let $h$ be the harmonic function on $Q(0,3)-\left[-\frac{1}{2}, \frac{1}{2}\right]$ with boundary values $h=1$ on $\partial Q(0,3)$ and $h=c_{1}$ on $\left[-\frac{1}{2}, \frac{1}{2}\right]$. Observe that

$$
h_{n}(z) \leq h\left(z / 3^{n-1}\right) \quad\left(z \in Q\left(0,3^{n}\right)\right) .
$$

Set $c=\sup _{z \in Q(0,1)} h(z)$. Obviously $c_{1}<c<1$. By (3), we see that $h_{n}(z) \leq c$ on $Q\left(0,3^{n-1}\right)$, and hence $h_{n}(z) \leq c h_{n-1}(z)$ on $Q\left(0,3^{n-1}\right)$. Therefore, (2) implies that

$$
h_{n}(x) \leq c c_{1} \quad\left(x \in\left[-3^{n-2} / 2,3^{n-2} / 2\right]\right) .
$$

From this, it follows that $h_{n}(z) \leq \operatorname{ch}\left(z / 3^{n-2}\right)$ on $Q\left(0,3^{n-1}\right)$, and hence, by the definition of $c$,

$$
h_{n}(z) \leq c^{2} \quad\left(z \in Q\left(0,3^{n-2}\right)\right) .
$$

Repeating this argument, we conclude that

$$
h_{n}(z) \leq c^{n} \quad(z \in Q(0,1)) .
$$

PROOF OF THEOREM 2. Let $\alpha=\frac{2}{3}$. For every $x \in \mathbf{R}$ with $3^{n+1} \leq|x| \leq 3^{n+2}$, choose $m \in \mathbf{Z}$ such that $|x-m| \leq \frac{1}{2}$. Since $Q\left(0,3^{n}\right)+m=Q\left(m, 3^{n}\right) \subset Q(x, \alpha|x|)$,

$$
\beta_{x}(z) \leq h_{n}(z-m) \quad\left(z \in Q\left(m, 3^{n}\right)\right) .
$$

With (1), this implies that

$$
\beta_{x}(x) \leq h_{n}(x-m) \leq c^{n} \quad\left(x \in \mathbf{R}, 3^{n+1} \leq|x| \leq 3^{n+2}\right) .
$$

Therefore,

$$
\begin{aligned}
\int_{|x| \geq 3} \frac{\beta_{x}(x)}{|x|} d x & =\sum_{n=0}^{\infty} \int_{3^{n+1} \leq|x| \leq 3^{n+2}} \frac{\beta_{x}(x)}{|x|} d x \\
& \leq 2 \sum_{n=0}^{\infty} \frac{c^{n}}{3^{n+1}} 3^{n+2}<\infty .
\end{aligned}
$$

By Benedicks' criterion I, this completes the proof.

REMARK. We take the Cantor ternary set for $E_{0}$ in Theorem 2 . Then, for the resulting Denjoy domain $D$, a boundary Harnack inequality yields that $\operatorname{dim} P_{p}(D)=$ 1 for every $p \in E-\{\infty\}$, however $\operatorname{dim} P_{\infty}(D)=2$ by Theorem 2 .

2. Throughout this section, suppose that $E$ is a compact set in $\mathbf{R}$ such that 0 is a point of density of $E$. We shall study the cardinal number $\operatorname{dim} P_{0}(D)$, where $D=\hat{\mathbf{C}}-E$.

Denote by $B(t, r)$ the disk $\{z ;|z-t|<r\}$. For an arbitrarily fixed $\delta \in\left(0, \frac{1}{3}\right)$ and every $x \in \mathbf{R}-\{0\}$, let $\gamma_{x}(\cdot)=\gamma_{x}(\cdot ; E, \delta)$ be the solution of Dirichlet problem on $B(x, \delta|x|)-E$ for boundary values $\gamma_{x}=1$ on $\partial B(x, \delta|x|)$ and $\gamma_{x}=0$ on $E \cap$ $B(x, \delta|x|)$. Choose $\alpha_{1}$ and $\alpha_{2}$ in $(0,1)$ such that $0<2 \alpha_{1}<\delta<\alpha_{2} /\left(2+\alpha_{2}\right)$. Consider the mapping $\phi(z)=1 / z$. Set $\omega_{x}^{i}=\beta_{1 / x}\left(\cdot ; \phi(E), \alpha_{i}\right), i=1,2$. Note that

$$
Q\left(1 / x, \alpha_{1} /|x|\right) \subset \phi(B(x, \delta|x|)) \subset Q\left(1 / x, \alpha_{2} /|x|\right) .
$$

Therefore, $\omega_{x}^{2}(1 / x) \leq \gamma_{x}(x) \leq \omega_{x}^{1}(1 / x)$, and hence, by Benedicks' criterion I, we obtain the following. 
BENEDICKS' CRITERION II. For every $\delta \in\left(0, \frac{1}{3}\right)$,

$$
\begin{aligned}
& \operatorname{dim} P_{0}(D)=1 \text { if and only if } \int_{|x| \leq 1} \frac{\gamma_{x}(x)}{|x|} d x=\infty \\
& \operatorname{dim} P_{0}(D)=2 \text { if and only if } \int_{|x| \leq 1} \frac{\gamma_{x}(x)}{|x|} d x<\infty
\end{aligned}
$$

We shall prove the following

THEOREM 4. Suppose that there exists $\lambda>\frac{1}{2}$ such that

$$
\frac{\left|E^{c} \cap I_{t}\right|}{t}=O\left(\left(\frac{1}{\log t^{-1}}\right)^{\lambda}\right)
$$

where $I_{t}=[-t, t]$. Then $\operatorname{dim} P_{0}(D)=2$.

Let $B$ be the unit disk $B(0,1)$. For $r \in(0,1]$, denote by $\Phi_{r}$ the class of harmonic functions $u$ such that (i) there exists a closed set $E_{u}$ in $[-1,1]$ with $\left|E_{u}\right| \geq 2(1-r)$, and (ii) $u$ is the solution of Dirichlet problem on $B-E_{u}$ for boundary values $u=1$ on $\partial B$ and $u=0$ on $E_{u}$. For the proof of Theorem 4, we need following lemmas.

LEMMA 5. Suppose that $u \in \Phi_{r}$. Then $u(t i)$ is an increasing function of $t \in$ $(0,1)$.

ProOF. We may assume that $E_{u}$ consists of finite number of closed intervals. Let $g_{B}$ be the Green's function on $B$ and set $\hat{g}(z, t i)=g_{B}(z, t i)+g_{B}(z,-t i)$, $0<t<1$. Applying Green's formula to $1-u$ and $\hat{g}(z, t i)$ on $B^{+}=B \cap\{\operatorname{Im} z>0\}$, we see that

$$
1-u(t i)=\frac{1}{2 \pi} \int_{-1}^{1} \hat{g}(x, t i)\left(\frac{\partial u}{\partial y}\right)_{y=0} d x
$$

where $z=x+i y$. Observe that $\hat{g}(x, t i),-1<x<1$, are decreasing functions of $t \in(0,1)$ and $(\partial u / \partial y)_{y=0} \geq 0$. Hence, by $(4), u(t i)$ is increasing on $(0,1)$.

LEMMA 6. For each $\rho \in[0,1)$, there exists a constant $C_{\rho}$ depending only on $\rho$ such that

$$
u(0) \leq C_{\rho} r^{\rho}, \quad 0<r \leq 1,
$$

where $u$ is any function in $\Phi_{r}$ such that 0 is a regular boundary point or an interior point of $B-E_{u}$.

Proof. Let $g^{+}$be the Green's function on $B^{+}=B \cap\{\operatorname{Im} z>0\}$. Since $g^{+}(z, w)=g_{B}(z, w)-g_{B}(z, \bar{w}), z=x+i y$,

$$
\left(\frac{\partial g^{+}(z, t i)}{\partial y}\right)_{y=0} \leq \frac{2 t}{x^{2}+t^{2}}, \quad 0<t<1 .
$$

Let $U$ be the harmonic function on $B^{+}$with boundary values $U=1$ on $\partial B^{+} \cap$ $\{|z|=1\}$ and $U=0$ on $\partial B^{+} \cap \mathbf{R}$. Observe that

$$
U(t i)=(4 / \pi) \tan ^{-1} t, \quad 0<t<1 .
$$


In order to show (5), it is sufficient to show that, for each $n \in\{0\} \cup N$, there exists a constant $C_{n}$ depending only on $n$ such that

$$
u(0) \leq C_{n} r^{1-2^{-n}}
$$

When $n=0,(8)$ is trivial. Suppose that (8) is valid for some $n$. For each $u \in \Phi_{r}$ and each $x \in\left[-\frac{1}{2}, \frac{1}{2}\right]$, there exists a $u_{x} \in \Phi_{r_{1}}, r_{1}=\min (1,2 r)$, such that $u(x+z / 2) \leq$ $u_{x}(z), z \in B$. Therefore,

$$
u(x) \leq u_{x}(0) \leq C_{n} r_{1}^{1-2^{-n}}=C_{n} r^{1-2^{-n}}
$$

for almost all $x \in\left[-\frac{1}{2}, \frac{1}{2}\right]$. Then, (6), (7), (9), and Green's formula yield that, for $t \in(0,1)$

$$
\begin{aligned}
u(t i) & =U(t i)+(u-U)(t i) \\
& =U(t i)+\frac{1}{2 \pi} \int_{-1}^{1} u(x)\left(\frac{\partial g^{+}(z, t i)}{t z \partial y}\right)_{y=0} d x \\
& \leq C t+\frac{1}{\pi}\left(\int_{-1 / 2}^{1 / 2} \frac{t u(x)}{x^{2}+t^{2}} d x+\int_{[-1,1]-[-1 / 2,1 / 2]} \frac{t u(x)}{x^{2}+t^{2}} d x\right) \\
& \leq C t+\frac{1}{\pi}\left(\frac{C_{n} r^{1-2^{-n}}}{t} \int_{[-1 / 2,1 / 2] \cap E_{u}^{c}} d x+4 \int_{E_{u}^{c}} d x\right) \\
& \leq C t+\frac{1}{\pi}\left(\frac{C_{n} r^{1-2^{-n}}}{t} 2 r+8 r\right) .
\end{aligned}
$$

Therefore, putting $t=r^{1-2^{-n-1}}$ and, by Lemma 5 , we have

$$
u(0) \leq u\left(r^{1-2^{-n-1}} i\right) \leq C_{n+1} r^{1-2^{-n-1}}
$$

if 0 is a regular boundary point or an interior point of $B-E_{u}$.

PROOF OF THEOREM 4. There exists a constant $C$ independent of $t$ such that

$$
\left|E^{c} \cap I_{t}\right| \leq C t /\left(\log t^{-1}\right)^{\lambda}, \quad 0<t \leq 1 .
$$

Then, for each $x$ in $\left(0, \frac{1}{2}\right]$,

$$
\frac{1}{\delta x}\left|E^{c} \cap[x-\delta x, x+\delta x]\right| \leq \frac{1}{\delta x} \frac{C(x+\delta x)}{\left(\log (x+\delta x)^{-1}\right)^{\lambda}} \leq \frac{C}{\left(\log x^{-1}\right)^{\lambda}} .
$$

Put $r=r_{x}=C /\left(2(\log (1 / x))^{\lambda}\right)$ and choose $t_{0} \in\left(0, \frac{1}{2}\right]$ such that $r_{x} \leq 1$ for each $x \in\left(0, t_{0}\right]$. Consider the function $u(z)=\gamma_{x}(x+\delta x z)$ on $B$. From (11), it follows that $u \in \Phi_{r_{x}}$ for each $x \in\left(0, t_{0}\right]$. Hence, by Lemma 6 , we can choose a $\rho \in(0,1)$ such that $\lambda \rho>\frac{1}{2}$ and

$$
\gamma_{x}(x)=u(0) \leq C_{\rho} r_{x}^{\rho}=C /\left(\log x^{-1}\right)^{\lambda \rho}
$$

for almost all $x$ in $\left(0, t_{0}\right]$, where $C$ is independent of $x$. Note that $\sigma=\lambda \rho+\lambda>1$. Setting $X(x)=\int_{E^{c} \cap[0, x]} d t$, by $(10)$,

$$
X(x) \leq C x /\left(\log x^{-1}\right)^{\lambda} .
$$


Write $t_{1}=\min \left(t_{0}, 1 / e^{\lambda \rho}\right)$. Since $\gamma_{x}(x)=0$ almost everywhere on $E$, using (12) and (13), we see that

$$
\begin{aligned}
\int_{0}^{t_{1}} \frac{\gamma_{x}(x)}{x} d x & \leq \int_{0}^{t_{1}} \frac{C}{x\left(\log x^{-1}\right)^{\lambda \rho}} d X \\
& =\frac{C X\left(t_{1}\right)}{t_{1}\left(\log t_{1}^{-1}\right)^{\lambda \rho}}-\int_{0}^{t_{1}} C X(x) d\left(\frac{1}{x\left(\log x^{-1}\right)^{\lambda \rho}}\right) \\
& \leq C+C \int_{0}^{t_{1}} \frac{x}{\left(\log x^{-1}\right)^{\lambda}} \frac{1}{x^{2}\left(\log x^{-1}\right)^{\lambda \rho}}\left(1-\frac{\lambda \rho}{\log x^{-1}}\right) d x \\
& \leq C+C \int_{0}^{t_{1}} \frac{d x}{x\left(\log x^{-1}\right)^{\sigma}}<\infty
\end{aligned}
$$

Similar argument yields that $\int_{[-1,0)}\left(\gamma_{x}(x) /|x|\right) d x<\infty$, and therefore the criterion II completes the proof.

3. In this section, we shall give an example of a compact subset $E$ of $\mathbf{R}$ such that

$$
\left|E^{c} \cap I_{t}\right| / t=O\left(\left(\log t^{-1}\right)^{-1 / 2}\right)
$$

and $\operatorname{dim} P_{0}(\hat{\mathbf{C}}-E)=1$, which shows that the condition $\lambda>\frac{1}{2}$ in Theorem 4 is, in a sense, best possible.

LEMMA 7. For $r$ in $(0,1)$, let $u_{r}$ be the harmonic function on $B-([-1,-r] \cup$ $[r, 1])$ with boundary values $u_{r}=1$ on $\partial B$ and $u_{r}=0$ on $[-1,-r] \cup[r, 1]$. Then there exists a constant $C$ independent of $r$ such that $u_{r}(0) \geq C r$.

PrOOF. Consider two functions $f(z)$ and $g(w)$ such that

$$
f(z)=\frac{\sqrt{(z+r) /(1+r z)}-\sqrt{r}}{1-\sqrt{r(z+r) /(1+r z)}} \cdot \frac{1+\sqrt{r(z+r) /(1+r z)}}{\sqrt{(z+r) /(1+r z)}+\sqrt{r}}
$$

and

$$
g(w)=\frac{\sqrt{(c-w) /(1-c w)}-\sqrt{c}}{1-\sqrt{c(c-w) /(1-c w)}} \cdot \frac{1+\sqrt{c(c-w) /(1-c w)}}{\sqrt{(c-w) /(1-c w)}+\sqrt{c}}
$$

where $c=f(r)$. Then, it is not difficult to see that $g(f(z))$ is the conformal mapping of $B-([-1,-r] \cup[r, 1])$ to $B$. Computing the arc length of $g(f(\partial B))$, we have

$$
u_{r}(0)=\frac{2}{\pi} \sin ^{-1} \frac{6 c-c^{2}-1}{(1+c)^{2}} \geq C r .
$$

Let $E=[-1,1]-\bigcup_{n=2}^{\infty} J_{n}$, where $J_{n}$ is the open interval $\left((1-1 / \sqrt{n}) e^{-n}\right.$, $\left.(1+1 / \sqrt{n}) e^{-n}\right), n=2,3, \ldots$ Then, for each $x \in J_{n}^{\prime}=\left((1-1 / 2 \sqrt{n}) e^{-n}\right.$, $\left.(1+1 / 2 \sqrt{n}) e^{-n}\right)$, there exists a constant $C$ independent of $n$ such that

$$
(1 / \delta x) \operatorname{dist}(x, E) \geq C / \sqrt{n} \text {. }
$$

By the definition of $u_{r}$ in Lemma 7, this means that $\gamma_{x}(x+\delta x z) \geq u_{r_{n}}(z), z \in B$, where $r_{n}=C / \sqrt{n}$. Hence, from Lemma 7 , it follows that

$$
\gamma_{x}(x) \geq u_{r_{n}}(0) \geq C / \sqrt{n}, \quad x \in J_{n}^{\prime} .
$$


Therefore,

$$
\begin{aligned}
\int_{0}^{1} \frac{\gamma_{x}(x)}{x} d x & \geq \sum_{n=2}^{\infty} \int_{J_{n}^{\prime}} \frac{\gamma_{x}(x)}{x} d x \\
& \geq \sum_{n=2}^{\infty} \frac{C e^{n+1}}{\sqrt{n}} \frac{e^{-n}}{\sqrt{n}}=\sum_{n=2}^{\infty} \frac{C}{n}=\infty .
\end{aligned}
$$

Thus the criterion II shows that $\operatorname{dim} P_{0}(\hat{\mathbf{C}}-E)=1$. On the other hand, it is easily seen that $\left|E^{c} \cap I_{t}\right| / t=O\left(\left(\log t^{-1}\right)^{-1 / 2}\right)$.

REMARK. It seems that the function $u_{r}$ in Lemma 7 satisfies that $u_{r}(0)=$ $\sup _{u \in \Phi_{r}} u(0)$, although we have not succeeded in the proof.

\section{REFERENCES}

1. A. Ancona, Une propriété de la compactification de Martin d'un domaine euclidien, Ann. Inst. Fourier (Grenoble) 29 (1979), 71-90.

2. M. Benedicks, Positive harmonic functions vanishing on the boundary of certain domains in $\mathbf{R}^{n}$, Ark. Mat. 18 (1980), 53-71.

3. C. Constantinescu and A. Cornea, Ideale Ränder der Riemannschen Flächen, Springer-Verlag; Berlin and New York, 1963.

4. J. B. Garnett and P. W. Jones, The Corona theorem for Denjoy domains, Acta Math. 155 (1985), 31-40.

Department of Mathematics, Daido institute of Technology, Daido, MiNAMI, NAGOYA 457 , JAPAN 